\title{
Dynamic Data Driven Applications Systems - DDDAS 2009
}

\author{
Craig C. Douglas \\ University of Wyoming, Laramie, WY 82071, USA \\ Yale University, New Haven, CT 06520-8285, USA
}

\begin{abstract}
This workshop covers several aspects of the Dynamic Data Driven Applications Systems (DDDAS) concept, which is an established approach defining a symbiotic relation between an application and sensor based measurement systems. Applications can accept and respond dynamically to new data injected into the executing application. In addition, applications can dynamically control the measurement processes. The synergistic feedback control-loop between an application simulation and its measurements opens new capabilities in simulations, e.g., the creation of applications with new and enhanced analysis and prediction capabilities, greater accuracy, longer simulations between restarts, and enable a new methodology for more efficient and effective measurements. DDDAS transforms the way science and engineering are done with a major impact in the way many functions in our society are conducted, e.g., manufacturing, commerce, transportation, hazard prediction and management, and medicine. The workshop will present such new opportunities as well as the challenges and approaches in technology needed to enable DDDAS capabilities in applications, relevant algorithms, and software systems. The workshop will showcase ongoing research in these aspects with examples from several important application areas.
\end{abstract}

\section{The Scope of the Workshop}

More and more applications are migrating to a data-driven paradigm including hazard management, terrorist event handling, contaminant tracking, chemical process plants, petroleum refineries, well bores, and nuclear power plants. In each case sensors produce large quantities of telemetry that are fed into simulations that model key quantities of interest. As data are processed, computational models are adjusted to best agree with known measurements. If properly done, this increases the predictive capability of the simulation system. This allows what-if scenarios to be modeled, disasters to be predicted and avoided with human initiated or automatic responses, and the operation of the plants to be optimized. As this area of computational science grows, a broad spectrum of application areas will reap benefits. Examples include enhanced oil recovery, optimized placement of desalination plants and other water intakes, optimized food production, monitoring the integrity of engineered structures and thus avoiding failures, and real time traffic advice for drivers. These are but a few of countless examples. 
As is the case in other data intensive arenas, visualization plays a key role in DDDAS. Visualization is used at all stages: setting up data and initial and/or boundary conditions, seeing and analyzing results, and steering computations.

Data-driven computational science is ripe for multidisciplinary research to build applications, algorithms, measurement processes, and software components from which tools can be developed to solve diverse problems of regional and international interest. The advances that will result, including enhanced repositories of software components and applications, will be of great value to industry and governments, and will set the stage for further valuable research and development. A comprehensive list of ongoing state of the art projects is kept up to date on http://www.dddas.org in the projects area.

Several research thrusts in which advances should significantly enhance the ability of data-driven computational science to bring its tremendous benefits to a wide array of applications. These research thrusts, which are described below in more detail, are:

- Effective assimilation of streams of data into ongoing simulations.

- Interpretation, analysis, and adaptation to assist the analyst and to ensure the most accurate simulation.

- Cyberinfrastructure to support data-driven simulations.

These three areas interact with two other research fields symbiotically: (1) forward multiscale modeling and simulation, and (2) deterministic and statistical methods in inverse problems.

Research areas (1) and (2) combined with (3) DDDAS must work within the context of uncertainty and will benefit from the development of statistically sound, unified treatments of uncertainties. For example, in forward multiscale modeling and simulation, input data are uncertain and these uncertainties should be propagated to uncertainties in output quantities of interest. In an inverse problem, proper treatment of measurement uncertainties and errors must be integrated with treatment of uncertainties associated with bforwardb models. be treated systematically. In a data-driven application, all of these uncertainties are present and must.

Data management in a DDDASis typically supported by tools for data acquisition, data access, and data dissemination. Data acquisition tools retrieve the real time or near real time data, processing and storing them. Data access tools provide common data manipulation support, e.g., querying, storing, and searching, to upper level models. Data dissemination tools read data from the data store, format them based on requests from data consumers, and deliver formatted data to data consumers.

Software re-use, supporting separation of concerns, and real time quality support for applications will increase reliability, reduce development time, and teach students how to be much more productive.

DDDAS and similar methodologies are the paradigm of the truly data rich information age upon us. 